\title{
Radiosensitization Effects by Bismuth Oxide Nanoparticles in Combination with Cisplatin for High Dose Rate Brachytherapy
}

This article was published in the following Dove Press journal: International Journal of Nanomedicine

\author{
Noor Nabilah Talik Sisin (D) \\ Khairunisak Abdul Razak (D) ${ }^{2}$ \\ Safri Zainal Abidin (1D ${ }^{3}$ \\ Nor Fazila Che Mat ${ }^{1}{ }^{\prime}$ \\ Reduan Abdullah (1D) 1,4 \\ Raizulnasuha Ab Rashid (D) \\ Muhammad Afiq Khairil \\ Anuar (D) \\ Nur Hamizah \\ Mohd Zainudin (iD) ${ }^{\prime}$ \\ Nashrulhaq Tagiling $\mathbb{D}^{\prime}$ \\ Norazlina Mat Nawi ${ }^{5}$ \\ Wan Nordiana Rahman (ID) \\ 'School of Health Sciences, Universiti \\ Sains Malaysia, Kubang Kerian, Kelantan, \\ Malaysia; ${ }^{2}$ School of Materials and Mineral \\ Resources Engineering, Universiti Sains \\ Malaysia, Nibong Tebal, Penang, Malaysia; \\ ${ }^{3}$ Advanced Medical and Dental Institute, \\ Universiti Sains Malaysia, Bertam, Penang, \\ Malaysia; ${ }^{4}$ Hospital of Universiti Sains \\ Malaysia, Kubang Kerian, Kelantan, \\ Malaysia; ${ }^{5}$ School of Medical Sciences, \\ Universiti Sains Malaysia, Kubang Kerian, \\ Kelantan, Malaysia
}

Correspondence: Wan Nordiana Rahman School of Health Sciences, Universiti Sains Malaysia, Kubang Kerian 16150, Kelantan, Malaysia

Tel +60 976778I I

Email wandiana@usm.my
Purpose: The aim of this study was to investigate the potential of the synergetic triple therapeutic combination encompassing bismuth oxide nanoparticles (BiONPs), cisplatin (Cis), and high dose rate (HDR) brachytherapy with Ir-192 source in breast cancer and normal fibroblast cell line.

Methods: In vitro models of breast cancer cell lines (MCF-7, MDA-MB-231) and normal fibroblast cell line (NIH/3T3) were employed. Cellular localization and cytotoxicity studies were conducted prior to inspection on the radiosensitization effects and generation of reactive oxygen species (ROS) on three proposed radiosensitizers: BiONPs, Cis, and BiONPs-Cis combination (BC). The optimal, non-cytotoxic concentration of BiONPs (0.5 $\mathrm{mM})$ and the $25 \%$ inhibitory concentration of Cis $(1.30 \mu \mathrm{M})$ were applied. The radiosensitization effects were evaluated by using a $0.38 \mathrm{MeV}$ Iridium-192 HDR brachytherapy source over a prescribed dose range of 0 Gy to $4 \mathrm{~Gy}$.

Results: The cellular localization of BiONPs was visualized by light microscopy and accumulation of the BiONPs within the vicinity of the nuclear membrane was observed. Quantification of the sensitization enhancement ratio extrapolated from the survival curves indicates radiosensitization effects for MCF-7 and MDA-MB-231 when treated with BiONPs, Cis, and BC. However, NIH/3T3 cells exhibited contradictive behavior as it only reacted towards the $\mathrm{BC}$ combination. Nonetheless, the MCF-7 cell line loaded with $\mathrm{BC}$ shows the highest SER of 4.29. ROS production analysis, on the other hand, shows that Cis and $\mathrm{BC}$ radiosensitizers generated the highest free radicals in comparison to BiONPs alone. Conclusion: A BiONPs-Cis combination was unveiled as a novel approach that offers promising radiosensitization enhancement that will increase the efficiency of tumor control while preserving the normal tissue at a reduced dose. This data is the first precedent to prove the synergetic implication of BiONPs, Cis, and HDR brachytherapy that will be beneficial for future chemoradiotherapy strategies in cancer care.

Keywords: HDR brachytherapy, bismuth oxide nanoparticles, radiosensitization, cisplatin, chemoradiotherapy

\section{Introduction}

Ionizing radiation emitted from sealed radiation source has started as a therapy in cancer care since the discovery of radium in $1898 .{ }^{1}$ High dose rate brachytherapy is one of the modalities that utilized sealed radiation source, ie iridium-192 to treat myriad types of malignancy such as breast, cervix, and prostate cancer. ${ }^{1}$ The estimates of the worldwide brachytherapy modalities in radiotherapy centers are around 34\% as reported by International Atomic Energy Agency (IAEA) in 2017. ${ }^{1}$ 
Brachytherapy is highly localized in comparison to external beam radiotherapy in which radioactive sources are delivered in proximity to the tumor sites internally and hence providing high radiation dose to the cancer cells while sparing the surrounding normal tissue. ${ }^{1,2}$

To improve the brachytherapy outcome, chemotherapy has been added to the treatment. The effects have shown to improve efficacy obtained with concurrent chemobrachytherapy as opposed to irradiation alone. ${ }^{3}$ Evidence from clinical trials has supported the advantages of concurrent chemobrachytherapy in local, regional control of the tumor. A study by Aghili et al indicates the feasibility of concurrent chemobrachytherapy in which the most observed side effects were grade 1 and 2, and deemed tolerable. ${ }^{4}$ Besides, few clinical studies also proved that the effect of the cisplatin and brachytherapy combination was compelling, deemed tolerable, and the percentage of disease-free survival after one year was more than $70 \%{ }^{3,5,6}$ The biological rationale is that a chemotherapy drug such as cisplatin could act as a radiosensitizer that can enhance radiation dose at the tumor site. In radiation therapy, radiosensitizers are applied to maximize the intrinsic radiosensitivity of cancer cells and increase radiation absorbed dose to the tumor site. Treatment could be performed with a lower radiation dose, which will reduce the harmful effects on normal cells.

Cisplatin or platinum diamino dichloride is one of the standard chemotherapeutic drugs used in clinical settings for various cancer treatments. Cisplatin is a platinumbased compound that has an atomic number of 78 may increase the probability radiation interaction that leads to the production of highly lethal low energy secondary electron. Cisplatin is also capable of generating free radicals that produce substantial DNA breakage. ${ }^{7}$ A fundamental study by Cho et al had determined the simulated dose enhancement effects by cisplatin, which justify the biological effects and support aforementioned the clinical observations. ${ }^{8}$ Cisplatin had also been tested on lung cancer cell lines with low dose fractionated radiation and increased the hyper-radiation sensitivity through clonogenic and apoptosis cell death mechanism. ${ }^{9}$

Evidence of survival improvements have been observed, but intrinsic toxicity remains a major issue with concurrent chemobrachytherapy. The potential benefit of concurrent chemoradiotherapy with HDR brachytherapy is, however, confined by the risk of complication due to the exposure of healthy organs to high dose rate radiation. Cisplatin also induced the formation of toxic platinum intermediates, which inhibit the post-irradiation DNA damage repairs, which could diminish normal cells' survival. ${ }^{10}$ To widen the therapeutic window of chemoradiotherapy, nanoparticles based radiosensitizer is introduced. In pre-clinical research, a few nanoparticle elements had shown the potential to be radiosensitizers, such as gold, superparamagnetic iron oxide, platinum, and bismuth nanoparticles. ${ }^{11-13}$ Gold nanoparticles have been widely investigated as a radiosensitizer for radiotherapy either alone or in combination with other compounds. Several studies had investigated dose enhancement of radiation with both cisplatin and gold nanoparticles. Cui et al had determined that gold nanoparticle would enhance cisplatin chemoradiation using small animal irradiator with dose enhancement factor (DEF) of 1.39 for MDA-MB-231 cells in vitro. ${ }^{7}$ Besides, Yang et al reported that the presence of peptide-modified gold nanoparticles and cisplatin during $6 \mathrm{MV}$ irradiation resulted in a $36 \%$ decrease in MDA-MB-231 cell survival when compared to chemoradiation alone. ${ }^{14}$ Another study on Monte Carlo simulation of brachytherapy using four different sources with gold nanoparticles and cisplatin produced DEF within the range of 1.03 to $3.43 .^{8}$

An alternative to gold nanoparticles, bismuth oxide nanoparticles (BiONPs) has been investigated as a potential radiosensitizer. ${ }^{13,15,16}$ The presence of bismuth may trigger additional retention, absorption, and scattering of the radiation at the cancer site, and thus demonstrated a higher enhancement of the dose, in comparison to other types of NPs. ${ }^{17,18}$ Increase in radiation interaction may occur due to the high atomic number of the bismuth element $(Z=83)$, which could instigate more photon absorption and release more electrons even when low radiation energy is being used. ${ }^{15,17,19}$ A study on radiosensitization of BiONPs, bismuth sulfide, and gold nanoparticles using 3D phantom demonstrated that all three nanoparticles could enhance the kilovoltage radiation effects with the DEF of BiONPs was the highest. ${ }^{20}$ GEANT4 was used to simulate brain tissue irradiation in the presence of bismuth oxide, and DEF quantified up to 18.55 which present promising results. ${ }^{15}$

In the present study, we selected bismuth oxide nanoparticles and cisplatin as an alternative radiosensitizer, which may potentially enhance the radiation dose through the synergetic effects of both compounds. The radiosensitization effects and generation of reactive oxygen species (ROS) induced by bismuth oxide nanoparticles (BiONPs) in combination with cisplatin (Cis), observed on MCF-7, 
MDA-MB-231 and NIH/3T3 cell lines for high dose rate (HDR) brachytherapy applications were investigated.

\section{Materials and Methods BiONPs Preparation}

The BiONPs were synthesized using a hydrothermal process and characterized according to the methods published in previous literature. ${ }^{19}$ The nanoparticles are in the monoclinic phase and rod shape with a diameter of $60 \mathrm{~nm}$ and length approximately $500 \mathrm{~nm}$. The BiONPs in powders form were diluted in complete culture media before usage.

\section{Cell Culture}

In this research, two breast cancer cell lines (MCF-7 and MDA-MB-231) and one normal fibroblast cell line (NIH/ 3T3), which commercially purchased from ATCC ${ }^{\circledR}$, were used in this study. All cells were cultured in Dulbecco's Modified Eagle's Medium (DMEM) supplied with 5\% fetal bovine serum and $1 \%$ of penicillin-streptomycin in the $25 \mathrm{~cm}^{2}$ flasks. The cells were cultured in an incubator, which was humidified using distilled water at a temperature of $37^{\circ} \mathrm{C}$ and $5 \% \mathrm{CO}_{2}$. All flasks were washed with phosphate buffer saline (PBS) once in two days until the cell confluency reached $90 \%$. The cells were detached using $0.25 \%$ trypsin-EDTA and prepared in suspension for the experiments.

\section{Cytotoxicity Assay of BiONPs}

MCF-7, MDA-MB-231, and NIH/3T3 were cultured in 96-wells plates with approximately 3000 cells in each well. The cells were incubated in a humidified environment of $37^{\circ} \mathrm{C}$ and $5 \% \mathrm{CO}_{2}$ until they reached about $80 \%$ confluency. The cells were washed with PBS before being treated with $60 \mathrm{~nm}$ BiONPs. The BiONPs in tubes were shaken vigorously or vortex for a few seconds to ensure the homogenous solution of BiONPs in the culture media before being introduced to the cells. The BiONPs were added into the wells with final concentrations of 0.00005 , $0.0005,0.005,0.05$ and $0.5 \mathrm{mmol} / \mathrm{l}(\mathrm{mM})$ and incubated for 24,48 and $72 \mathrm{hrs}$. The cytotoxicity effects were evaluated by measuring cell proliferation using Presto Blue ${ }^{\mathrm{TM}}$ Cell Viability Reagent (Life Technologies, California, United States). The Presto Blue ${ }^{\mathrm{TM}}$ reagent was added to the cells with a ratio of 9:1 of media to the reagent. After $4 \mathrm{hrs}$ incubation, the Presto Blue ${ }^{\mathrm{TM}}$ reactions with cells were measured using a microplate reader (Varioskan Flash, ThermoFisher Scientific, United States) in fluorescence mode (excitation wavelength of $535 \mathrm{~nm}$, the emission wavelength of $615 \mathrm{~nm}$ ). The optimal non-toxic BiONPs concentration was determined by taking the concentration that produces the cell viability of more than $80 \%$ after treatment.

\section{Cytotoxicity Assay of Cisplatin}

Evaluations of cytotoxic effects of cisplatin on MCF-7, MDA-MB-231, and NIH/3T3 cells were conducted by plating approximately 7500 cells per well in 96 wells plates and incubated until the cells reached $80 \%$ confluency. Cisplatin was prepared as $10 \mathrm{mg} / \mathrm{mL}$ stock solution, and two-serial dilution was conducted to yield dilutions ranging from 0.39 to $100 \mu \mathrm{g} / \mathrm{mL}$ (1.03 to 333 $\mu \mathrm{M}) .2 \mu \mathrm{L}$ of each dilution was added into $200 \mu \mathrm{L}$ fresh media in each well. The treated cells were incubated for $48 \mathrm{hrs}$ before the cell viability was quantified using Presto Blue $^{\text {TM }}$ reagent. Similar to cytotoxicity test of BiONPs, the cells were incubated with Presto Blue ${ }^{\mathrm{TM}}$ reagent for $4 \mathrm{hrs}$ before the fluorescence measurement was conducted using the microplate reader. The optimal concentration was determined from the dose-response curve fit at $75 \%$ cell viability $\left(\mathrm{IC}_{25}\right)$.

\section{Cellular BiONPs Localization}

The cellular localisations of BiONPs were done by plating approximately $1 \times 10^{5}$ cells on a sterile $22 \times 22 \mathrm{~mm}$ of size glass coverslip in a 6-well plate. The BiONPs of $60 \mathrm{~nm}$ size were added to the cell culture when the cells were grown to $80 \%$ confluency. The final concentration of BiONPs in each well was $0.5 \mathrm{mM}$. After $24 \mathrm{hrs}$ incubation, the cells were washed twice with PBS to rinse out the remaining BiONPs outside the cells. Then the cells fixed with cold methanol for 30 mins and stained with crystal violet for $1 \mathrm{hr}$. The cells samples were then washed and left to dry. The glass coverslips were mounted with 1 drop of dibutyl phthalate polystyrene xylene (DPX) on glass slides. The slides were observed under a light microscope at $100 \times$ magnification.

\section{Cells Irradiation Set Up}

The cells were irradiated in suspension with 1 x $10^{5}$ cells in $0.2 \mathrm{~mL}$ of Eppendorf tubes. Treatment with individual BiONPs, Cis, and BiONPs-Cis combination (BC) were conducted $2 \mathrm{hrs}$ prior to the irradiation. The treatment components details are presented in Table 1. Irradiations were performed with $0.38 \mathrm{MeV}$ of gamma-ray emitted from Iridium-192 source using Microselectron HDR 
Table I Treatment Components for the Radiosensitization Effect Study

\begin{tabular}{|l|l|}
\hline Treatment & Components \\
\hline Radiation dose (Gy) & 0 (positive control), I, 2, 3, 4 \\
Negative control & Untreated with BiONPs or Cis \\
Radiation + BiONPs & Radiation + 0.5 mM BiONPs \\
Radiation + Cis & Radiation + I.30 $\mu \mathrm{M}$ Cis \\
Radiation + BC & Radiation + 0.5 mM BiONPs + I.30 $\mu \mathrm{M}$ Cis \\
\hline
\end{tabular}

Abbreviations: cGy, centigray; BiONPs, bismuth oxide nanoparticles; Cis, cisplatin; $\mathrm{BC}, \mathrm{BiONPs}-\mathrm{Cis}$ combination.

Brachytherapy V14.23 system (Nucletron Corp, Columbia, Maryland). The irradiation was performed at Nuclear Medicine, Radiotherapy and Oncology Department, Hospital of Universiti Sains Malaysia. The cell samples were set up on top of water equivalent phantom between the surface mold and bolus to achieve the uniform dose. The cells were irradiated with radiation dose of 0 to 400 cGy in single fraction at room temperature. Gafchromic EBT3 films were used to validate the uniformity of the dose to the cell samples across the irradiation field.

\section{Clonogenic Survival Assay}

After irradiation, the cells were seeded into 6-wells plate, and complete media were added. The cells were incubated for 5 days for MCF-7 cells and NIH/3T3 cells, and 10 days for MDA-MB-231 cells in which the cells colonies were formed correctly. Then, the cells were fixed with cold methanol and stained with crystal violet. The image of colony formation on plates was analyzed using ImageJ software to virtually count and scored the stained cells colonies. Survival fractions were calculated and are represented by the ratio of colony formation after exposure to radiation to those unexposed to radiation.

\section{Reactive Oxygen Species Measurement}

Approximately $5 \times 10^{4}$ cells per $\mathrm{mL}$ were seeded into the 96-well plate and incubated overnight. The next day, the old media was discarded and fresh media were added, together with the treatment components (BiONPs, Cis, or BC). $100 \mu \mathrm{M}$ of dichlorodihydrofluorescein-diacetate (DCFH-DA) reagent were added into each well to a final concentration of $9 \mu \mathrm{M}, 1 \mathrm{hr}$ prior to irradiation with doses of 0,3 and $6 \mathrm{~Gy}$. The ROS generation was measured through the detection of $2^{\prime}, 7^{\prime}$-dichlorofluorescein (DCF) within the cells. The ROS were measured under fluorescence mode using microplate reader, immediately before and immediately after irradiation was performed.

\section{Cell Survival Analysis}

The cell survivals were analyzed by calculating the survival fraction from the cell colonies according to Equation 1.

$$
\text { Survival fraction }=\frac{\text { Number of irradiated cell colonies }}{\text { Number of control cell colonies }}
$$

The survival fractions for samples with and without (BiONPs, Cis, or BC) were then plotted and fitted according to the linear-quadratic (LQ) model, a gold standard to fit survival curves applied in clinical radiotherapy, using OriginPro 2018 software. The parameters from the LQ formula given by Equation 2 were analyzed.

$$
\mathrm{S}=\exp ^{-(\alpha \mathrm{D}+\beta \mathrm{D} 2)}
$$

In Equation 2, $\mathrm{S}$ is the survival fraction and $\mathrm{D}$ is the dose in Gray. The model represented by the linear component $[\exp (-\alpha \mathrm{D})]$ and quadratic component $\left[\exp \left(-\beta \mathrm{D}^{2}\right)\right]$ where $\alpha$ indicate single hit double-strand break of two chromosomes and $\beta$ indicate double hits that induce double-strand break of two chromosomes.

\section{Quantification of Radiosensitization Effects}

The experimental radiosensitization effects from the cell survival curves were measured using the sensitization enhancement ratio (SER) that indicates an enhancement of radiation effects by BiONPs and Cis in cells. The SER was extrapolated from the cell survival curves and calculated by taking the ratio of dose that produces $50 \%$ of cell survival fraction for control cells to treated cells as depicted in Equation 3.

$$
S E R_{50}=\frac{D_{50, \text { control }}}{D_{50, \text { BiONP }}}
$$

Increases in the radiation effects are commensurable to the increase in radiation dose. Radiation dose enhancement factors (DEF) can be theoretically predicted from the calculation of the ratio of the mass-energy absorption coefficient of the Cis and BiONPs to water, as shown in Equation 4.

$$
\begin{aligned}
D E F & =\frac{\left[\frac{\mu_{e n}}{\rho}\right]_{E}^{\text {water }+N P S}}{\left[\frac{\mu_{e n}}{\rho}\right]_{E}^{\text {water }}} \\
& =\frac{w_{N P S} \cdot\left[\frac{\mu_{e n}}{\rho}\right]_{E}^{N P S}+\left(1-W_{N P S}\right) \cdot\left[\frac{\mu_{e n}}{\rho}\right]_{E}^{\text {water }}}{\left[\frac{\mu_{e n}}{\rho}\right]_{E}^{\text {water }}}
\end{aligned}
$$

Determination of the theoretical dose enhancement factors requires the effective atomic number $\left(\mathrm{Z}_{\mathrm{eff}}\right)$ values, which are calculated using Equation 5: 


$$
Z_{\text {eff }}=\sqrt[m]{\sum^{f_{i}} Z_{i}^{m}}
$$

where $\mathrm{m}$ is the exponent of 2.94 , and $\mathrm{f}$ is the fraction of each element to the total electron number in each composite [1]. The estimated $\mathrm{Z}_{\text {eff }}$ of $\mathrm{Cis}$ and BiONPs were used as references to obtain the mass energy-absorption coefficient $\left[\frac{\mu_{e n}}{\rho}\right]_{E}$ for each compound from the physical data references of the National Institute of Standard and Technology database. ${ }^{21}$ The calculated $Z_{\text {eff }}$ of Cis and BiONPs is generally closer to samarium $(Z=62)$ and platinum $(\mathrm{Z}=78)$, respectively.

\section{Statistical Analysis}

All the data presented are expressed as the mean \pm standard error of the mean of the samples. All tests and graphs were done using OriginPro 2018 software.

\section{Results}

\section{Theoretical Calculation for DEFs}

The variation of the theoretical DEF caused by BiONPs and $C$ is against radiation energies for a range of 0.001 to $20 \mathrm{MeV}$ monoenergetic photon beam were presented in Figure 1. The highest DEF of 8.58 is obtained with the BC component at $0.05 \mathrm{MeV}$. Subsequently, the highest DEF for BiONPs and $\mathrm{Cis}$ are 4.51 at $0.04 \mathrm{MeV}$, and 5.70 at 0.06 $\mathrm{MeV}$, respectively. The DEF gradually decreased when approaching $1 \mathrm{MeV}$ and started to increase again at 3 $\mathrm{MeV}$. DEF is clearly dependent on the beam energy and

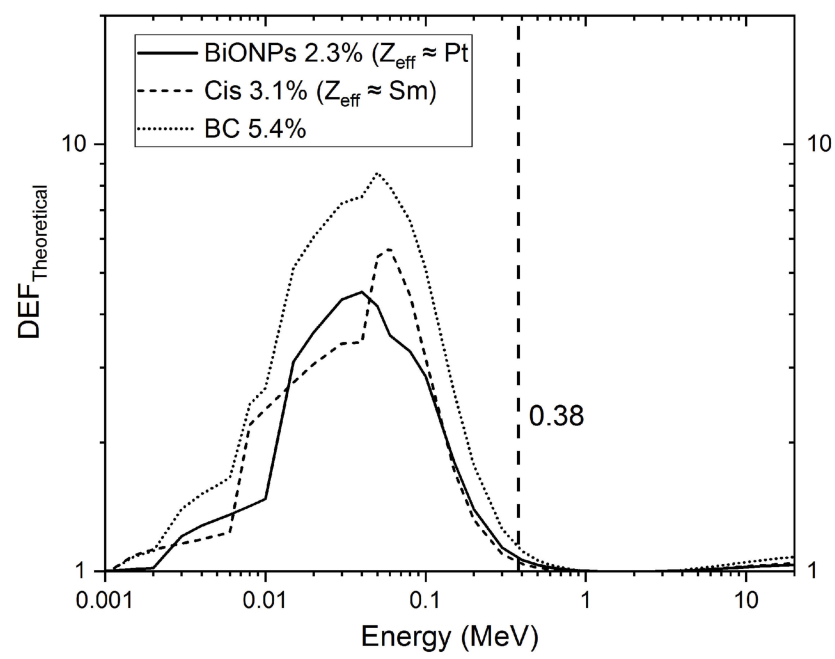

Figure I Theoretical dose enhancement factor (DEF) of each component at various energy ranges, with brachytherapy range at $0.38 \mathrm{MeV}$.

Notes: DEF of $\mathrm{Cis}$ and BiONPs is interpolated using the $\mathrm{Sm}$ and $\mathrm{Pt}$ database. The percentage of each component depended on the volume used during the irradiation.

Abbreviations: Zeff, effective atomic number; Sm, Samarium; Pt; platinum. the effective atomic number $\left(\mathrm{Z}_{\mathrm{eff}}\right)$. For 192-Ir HDR brachytherapy with the energy of $0.38 \mathrm{MeV}$, the DEF attained were 1.06, 1.04, and 1.12 for BiONPs, Cis, and $\mathrm{BC}$ respectively. $\mathrm{BC}$ is predicted to produce the highest radiosensitization effects in comparison to BiONPs and cisplatin alone.

\section{Cytotoxicity of BiONPs}

The BiONPs with a diameter size of $60 \mathrm{~nm}$ were tested on MCF-7, MDA-MB-231, and NIH/3T3 to determine the optimal non-toxic BiONPs concentration. Figure 2 present percentage of cell viability for concentration 0.00005 , $0.0005,0.005,0.05$ and $0.5 \mathrm{mM}$ of BiONPs incubated with the cells for 24,48 and $72 \mathrm{hrs}$. The results show that treatments with all concentrations of BiONPs indicate the percentages of cell viability are over $80 \%$, which are considered non-toxic for all three types of cell lines. Thus, the highest concentration of $0.5 \mathrm{mM}$ was regarded as an appropriate and suitable concentration to be used in the radiosensitization study without inducing unwanted reaction to the cells.

\section{Localization of BiONPs}

The BiONPs' cellular localisations were observed by staining the cells with crystal violet resulting in deep purple color of the cells' nuclei. Figure 3 shows the cells' image in which the locations of the rod-shaped BiONPs are visually visible under the microscope. The BiONPs are considered to be internalized into the cells as the microscopically observed nanoparticles are found to be confined within the cellular membranes and especially accumulated at the cells' cytoplasm surrounding the nuclei, particularly for smaller size cells which are MDA-MB-231 and MCF-7 (Figure 3A and B). In a bigger size of cells such as NIH/ 3T3, some of the BiONPs could also be seen scattered within the cytoplasm of the cells (Figure 3C).

\section{Cytotoxicity of Cisplatin}

The compatible concentration of cisplatin was determined according to $\mathrm{IC}_{25}$, the concentration that causes $25 \%$ cell inhibition to reduce the side effects of the drug and emphasize the reaction of BiONPs. In Figure 4, the concentrations that cause $75 \%$ cell viability $\left(\mathrm{IC}_{25}\right)$ by the cisplatin were identified from the curve fit as 10.72 , 1.30 , and $14.96 \mu \mathrm{M}$ for MCF-7, MDA-MB-231, and NIH/3T3 cells respectively. The graphs in Figure 4 demonstrate the cisplatin exerted a distinct effect on different cells. The cisplatin caused the highest toxicity 

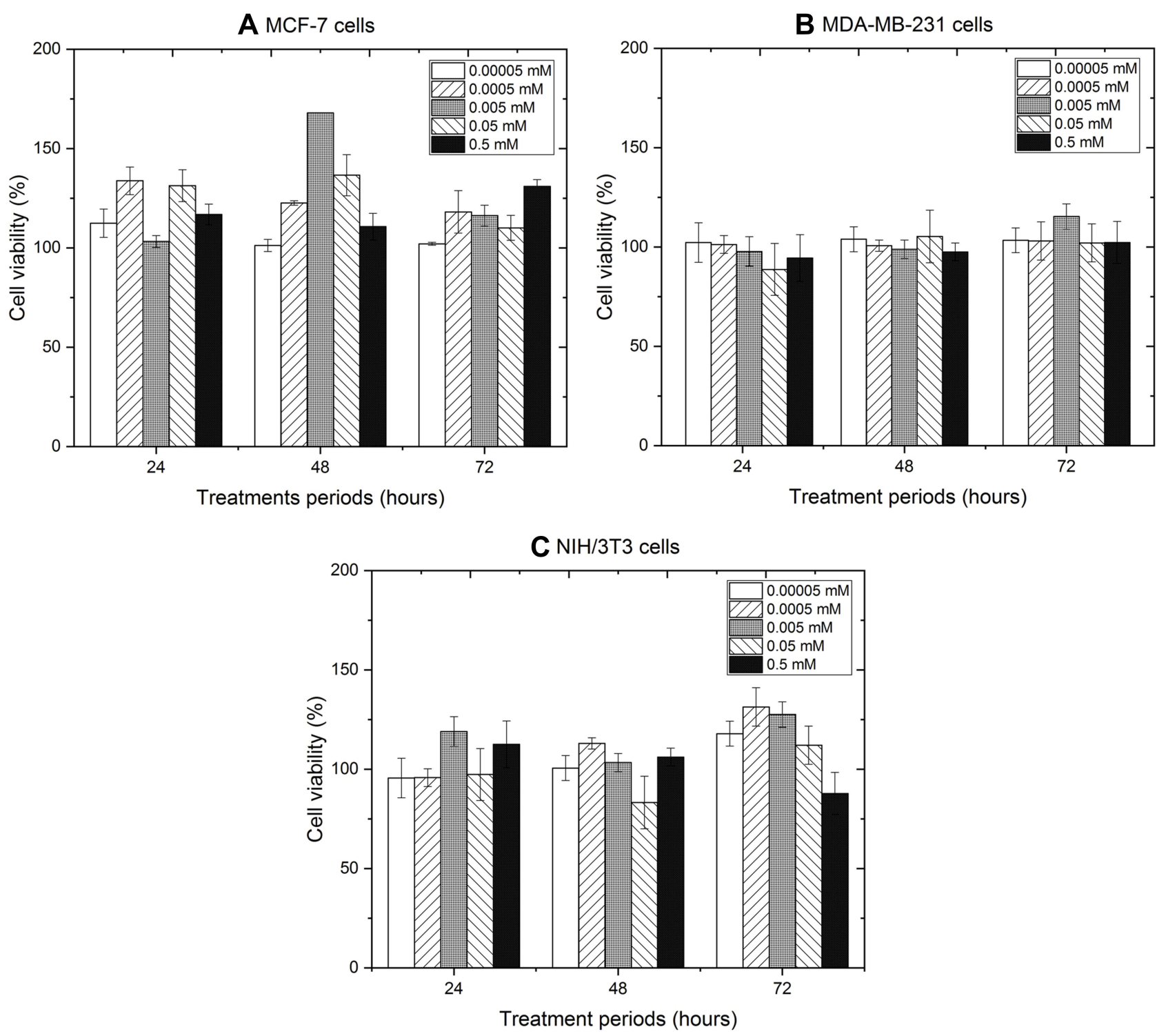

Figure 2 Percentage of cell viability after treatment with $60 \mathrm{~nm}$ BiONPs of different concentrations for 24, 48, and 72 hrs, on (A) MCF-7, (B) MDA-MB-23I and (C) NIH/ 3 T3 cells.

Notes: Error bars represent the standard error of the mean (SEM).

Abbreviation: BiONPs, bismuth oxide nanoparticles.

towards MDA-MB-231 breast cancer cells, followed by MCF-7 breast cancer cells, and NIH/3T3 normal fibroblast cells. The lowest $\mathrm{IC}_{25}$ value $(1.30 \mu \mathrm{M})$ was chosen to be a component of treatment in the irradiation.

\section{Radiosensitization Effects}

Survival curves of the MCF-7, MDA-MB-231, and NIH/ 3T3 irradiated by ${ }^{192}$ Ir HDR brachytherapy for control, BiONPs, $\mathrm{Cis}$ and $\mathrm{BC}$ is displayed in Figure 5. Radiosensitization effects depicted by the survival curve are clearly observed for MCF-7 and MDA-MB-231 cells, which show lower survival in the presence of the BiONPs,
Cis, and BC. The NIH/3T3 cells survival, however, does not show any reduction of survival except for BC combination. Quantitative evaluation of sensitization enhancement ratio (SER) in the BiONPs, Cis, and $\mathrm{BC}$ presence for MCF7 cells show SER value of $1.67,1.70$ and 4.29 respectively. Both of the radioresistant cells present less SER value of 1.03, 1.22 and 1.41 for MDA-MB-231 cells and 0.77, 0.66 and 1.65 for NIH/3T3 cells respectively for BiONPs, Cis, and BC. Neither BiONPs nor Cis alone could induce radiosensitization in NIH/3T3 cells, and only BC is able to effectuate radiosensitization effects in all types of cells. All the SER values are tabulated in Table 2. 

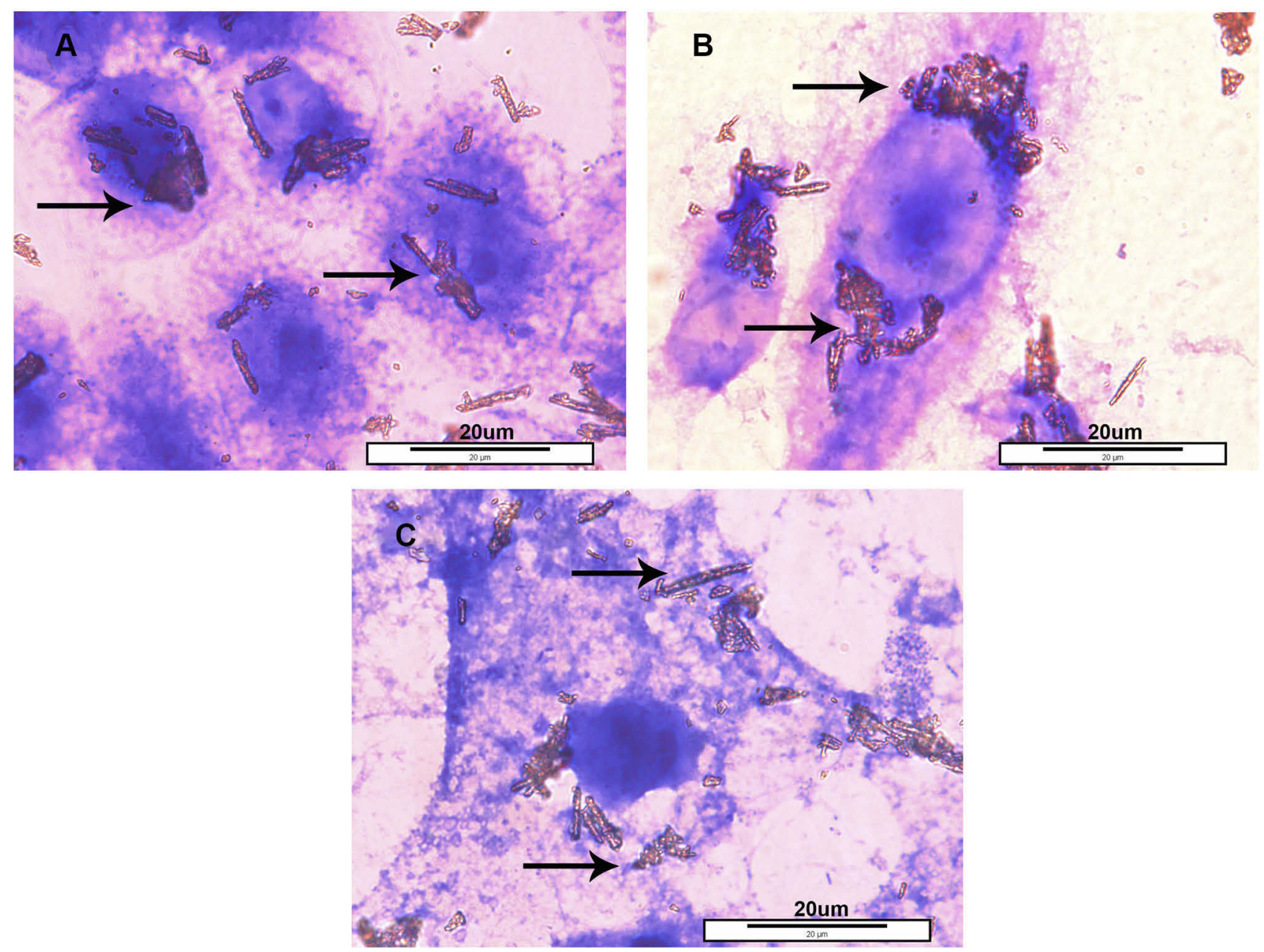

Figure 3 Localisation of BiONPs near the nuclei inside the (A) MCF-7, (B) MDA-MB-23I and (C) NIH/3T3 cells, after 24 hrs of incubation with 0.5 mMol/L of the BiONPs. Notes: Arrows indicated the rod-shaped BiONPs. The scale of each picture is $20 \mu \mathrm{m}$.

Abbreviation: BiONPs, bismuth oxide nanoparticles.

Radiobiological analysis of the alpha $(\alpha)$ and beta $(\beta)$ value from the data computed from the linear-quadratic model of cell survival data are also summarized in Table 2 . The $\alpha$ values associated with double-strand breaks for BC are found to be higher than the control cells and cells treated with BiONPs or Cis alone in MCF-7 and NIH/T3 cells. This might indicate $\mathrm{BC}$ is more potent for tumor cell killing in both cells. The experimental results are in agreement with the calculated theoretical DEF that shows $\mathrm{BC}$ produces the highest radiosensitization. Figure 6 shows the comparison of theoretical DEF and experimental SER obtained for MCF-7, MDA-MB-231, and NIH/3T3 cell lines.

\section{ROS Measurements After Irradiation}

The intracellular ROS generation was evaluated before and immediately after irradiation was conducted. Figure 7 shows the increase of ROS percentage when the cells underwent the respective treatments in comparison to control (untreated cells and without radiation). Generally, the ROS level after irradiation was lower in NIH/3T3 cells than in MCF-7 and MDA-MB-231. The production of ROS is also dependent on the radiation doses. When treated with higher doses together with different components, the cells elicited a higher amount of ROS. The presence of $\mathrm{Cis}$ and $\mathrm{BC}$ has been found to further heighten the generation of intracellular ROS, in comparison to the presence of BiONPs only.

\section{Discussion}

Cytotoxicity of BiONPs of $60 \mathrm{~nm}$ diameter of different concentrations was tested on MCF-7, MDA-MB-231 (breast cancer), and NIH/3T3 (normal) cell lines. The percentage of viable cells across treatment intervals 24 , 48 , and $72 \mathrm{hrs}$ are found to be more than $80 \%$ for all concentrations from 0.00005 to $0.5 \mathrm{mM}$. The highest 


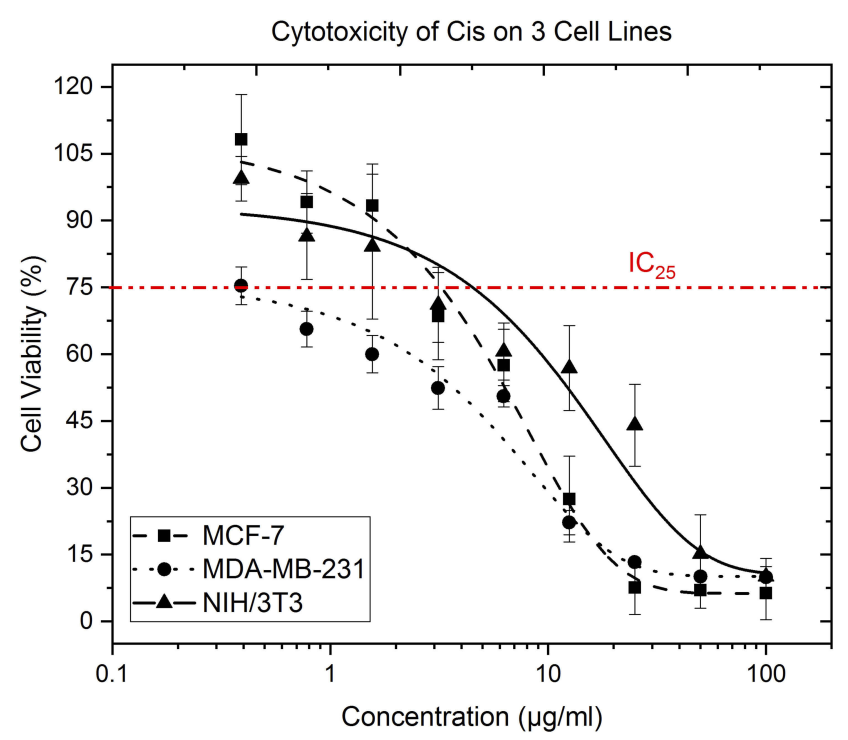

Figure 4 Cytotoxic evaluation of cisplatin against MCF-7, MDA-MB-23I, and NIH/ 3T3 cell lines. Each point shows the percentage of viable cells in comparison to the negative control. Curves are fitted using the Dose-Response model.

Notes: Error bars represent the standard error of the mean (SEM).

concentration of BiONPs used, which is $0.5 \mathrm{mM}$ could be considered as non-toxic and suitable to be applied as radiosensitizer due to the high percentage of cell viability after the treatment on all cell lines. However, in contrast to the present finding, cytotoxic response studies from previous reports that tested BiONPs of concentration around $0.215 \mathrm{mM}(100 \mu \mathrm{g} / \mathrm{mL})$ exhibited results of cell viability less than $80 \% .^{22,23}$ Temperature during the synthesis process could be one of the factors that influence cytotoxicity. $^{22}$ The nanoparticles' cytotoxicity also could be affected by the size of the NPs produced as the $97 \mathrm{~nm}$ of spherical BiONPs cause $73 \%$ of MCF-7 viable cells, while the $300 \mathrm{~nm}$ of rod-shaped BiONPs resulted in only $63 \%$ of Chang liver cell viability. ${ }^{22,23}$ The current inference is also in agreement with a prior work, which revealed that $60 \mathrm{~nm}$ rod-shaped BiONPs are less toxic than $90 \mathrm{~nm}$ BiONPs of the same shape. ${ }^{19}$ Thus, it is confirmed that $0.5 \mathrm{mM}$ of $60 \mathrm{~nm}$ BiONPs utilized the present study is biocompatible in vitro even in extended exposure.

The discovery of nanoparticle localization is one of the steps in determining the mechanism of radiation enhancement due to nanoparticle presence. Qualitative observation of the nanoparticles by using crystal violet dye that stained the proteins and DNA of the cells in the nuclei provides the visual location of the BiONPs inside the cells. ${ }^{24}$ The BiONPs have been observed surrounding the nuclei or scattered in the cytoplasm, which proves BiONPs penetration into the cells. In our recently published work, the BiONPs uptake into the cells was demonstrated quantitatively using flow cytometry gating, in which the high Q2 quadrant percentage for treatment of BiONPs onto the cells indicated the cellular uptakes of BiONPs. ${ }^{25}$ Theoretically, the BiONPs could enter the cells through macropinocytosis pathway, which is a cell transport system with a minimum of $0.5 \mu \mathrm{m}$ vesicular size. ${ }^{26}$ Nevertheless, the BiONPs could not enter the nucleus, especially the nuclear membrane. These findings are also in agreement with previous studies by Kim et al and Stewart et al, which deduced that nanoparticles of a size larger than $50 \mathrm{~nm}$ were unlikely able to enter the nucleus. ${ }^{16,27}$ The nuclear pore complex (NPC) at the nuclear membrane might be the reason in which the NPC size for MCF-7, MDA-MB-231 and NIH/3T3 might be less than BiONPs size of $60 \mathrm{~nm}$ with length up to $500 \mathrm{~nm}$ and hence does not permit the BiONPs to traverse into the nucleus.

This study introduced the BiONPs and the chemotherapeutic drug Cis, individually and in combination, during the radiotherapy, described as chemoradiotherapy study. Based on Table 2, it is perceived that the radiosensitization is enhanced in cancer cells compared to healthy cells, in the presence of either BiONPs or Cis. This occurrence indicated that both components are good candidates for radiosensitizers in cancer treatments. However, there are also major enhancement differences between cancer cell lines, MCF-7, and MDA-MB-231. Different types of receptors and different miRNA expression found in each cell line might be the reasons behind the lower sensitization enhancement in MDA-MB-231 cells. There were also 46 exclusive microRNAs in MDA-MB-231 cells, absent in MCF-7 cells. ${ }^{28}$ These microRNAs might originate the resistance of the MDA-MB-231 cells towards the personal effects of BiONPs and Cis. MCF- 7 cells are known to be estrogen receptor (ER) positive, while MDA-MB-231 cells are ER, progesterone receptor (PR) and human epidermal growth factor receptor 2 (HER2) negatives. ${ }^{28,29}$ The existence of the receptors influenced the molecular subtypes and the growth rate of the cells. Moreover, MDA-MB-231 cells are classified as basal-like cells subtypes, and their properties mirrored the characteristics of the normal cells. ${ }^{29}$ This statement is in agreement with the present study that individual BiONPs and $\mathrm{Cis}$ did not enhance the radiosensitization in both MDA-MB-231 and NIH/3T3 cells.

On the other hand, the combination of $\mathrm{BC}$ components had shown sensitization enhancement in all three cells. These results indicate that the triple combination of $\mathrm{BC}$ 

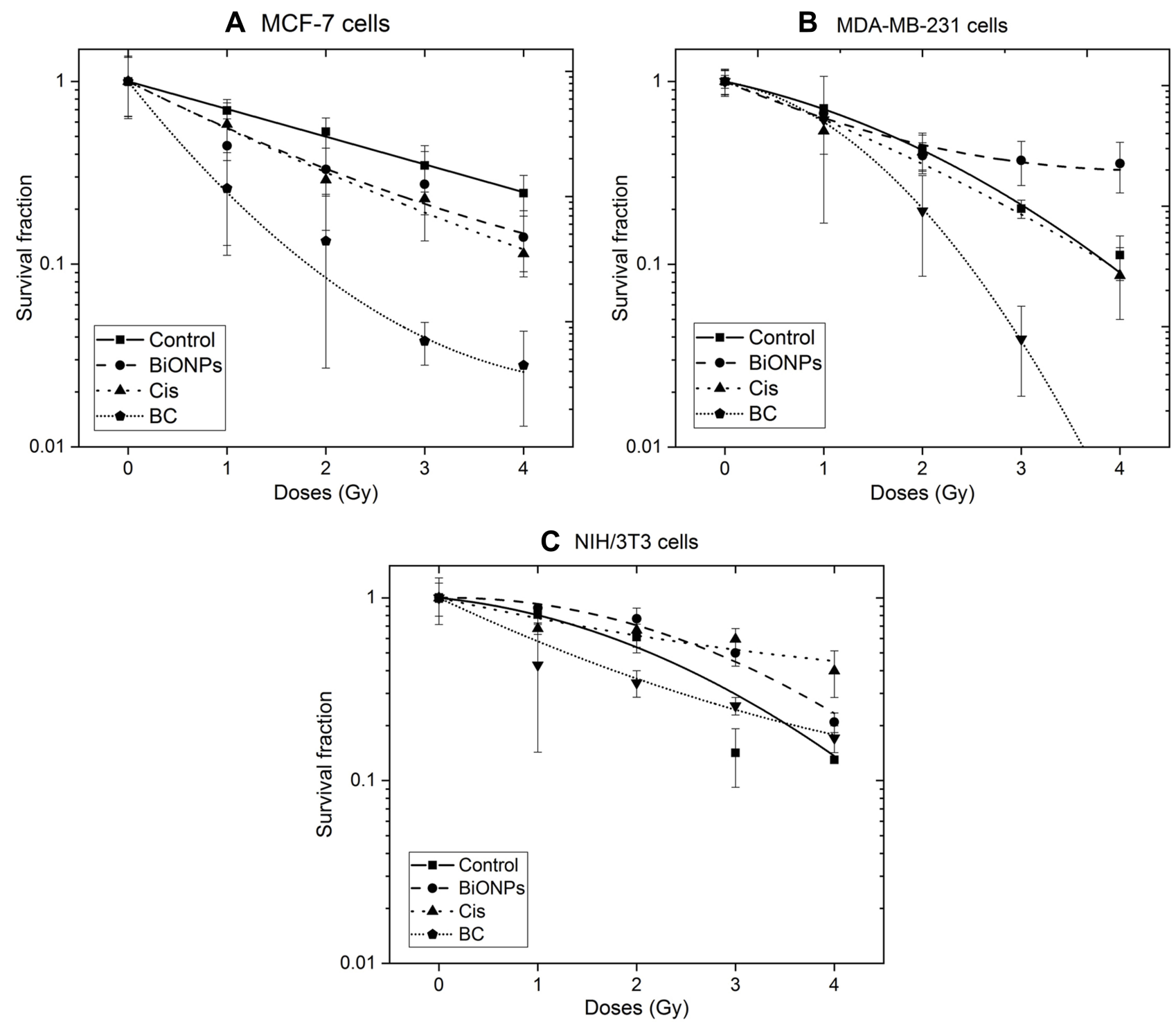

Figure 5 Survival curves of control, BiONPs, Cis, and BC treated on (A) MCF-7, (B) MDA-MB-23I and (C) NIH/3T3 cells irradiated with HDR brachytherapy. The survival data were fitted to linear-quadratic (LQ) models.

Notes: Error bars represent the standards errors of survival fractions.

with radiotherapy is more potent than the double combination of neither BiONPs nor Cis individually with radiation. The mechanism of the enhancement may involve synergistic effects between the enhancement caused by both BiONPs and Cis, as hypothesized by Eblan and Wang. ${ }^{30}$ In other studies involving the combination of an additional compound with $\mathrm{Cis}$, it was mentioned that the compound might either contribute to enhance the cell death triggered by $\mathrm{Cis}$ or it is the $\mathrm{C}$ is that amplified the effect of the compound. ${ }^{7,31}$ In the present study, only $\mathrm{IC}_{25}$ of the $\mathrm{Cis}$ $(1.30 \mathrm{uM})$ was used to lessen the drug effect and increase the impact of the BiONPs. Subsequently, any process transpire among the cells might be due to the dynamism of BiONPs, while being boosted by the Cis. Also, it can be seen that the SER value of MCF-7 cells is three to fourfold higher than MDA-MB-231 cells and NIH/3T3 cells. The findings signify that the presence of $\mathrm{BC}$ combination as a radiosensitizer combination. Survival curves were fitted with the LQ model, describing $\alpha$ and $\beta$ parameters as single and double hits of DNA breakages, respectively. From the results, it has been shown that all the BC combination treatments depicted higher $\alpha$ values, which portrays a large number of single hit occurring at the initial doses range. This finding shows that $\mathrm{BC}$ combination could be a suitable treatment approach compared to regular conventional treatment. During the radiation treatment 
Table 2 Radiobiological Analysis Based on Linear Quadratic Models Corresponding to Figure 6

\begin{tabular}{|l|l|l|l|l|l|}
\hline Cell & & Control & BiONPs & Cis & BC \\
\hline MCF-7 & $\alpha$ & $0.35 \pm 0.02$ & $0.62 \pm 0.13$ & $0.62 \pm 0.10$ & $-0.02 \pm 0.03$ \\
& $\beta$ & $0.00 \pm 0.01$ & $-0.04 \pm 0.04$ & 1.70 & $1.56 \pm 0.14$ \\
& SER & 1.00 & 1.67 & $0.16 \pm 0.04$ \\
\hline MDA-MB-23I & $\alpha$ & $0.26 \pm 0.03$ & $0.52 \pm 0.07$ & $0.44 \pm 0.13$ & 4.29 \\
& $\beta$ & $0.08 \pm 0.01$ & $-0.06 \pm 0.02$ & $0.04 \pm 0.04$ & $0.22 \pm 0.02$ \\
& SER & 1.00 & 1.03 & 1.22 & $0.29 \pm 0.01$ \\
\hline NIH/3T3 & $\alpha$ & $0.12 \pm 0.15$ & $-0.02 \pm 0.06$ & $0.27 \pm 0.10$ & 1.41 \\
& $\beta$ & $0.09 \pm 0.06$ & $0.10 \pm 0.02$ & $-0.02 \pm 0.03$ & $0.59 \pm 0.06$ \\
& SER & 1.00 & 0.77 & 0.66 & $-0.04 \pm 0.02$ \\
\hline
\end{tabular}

Notes: Control represents radiation treatment only.

Abbreviations: SER, sensitization enhancement ratio; cGy, centigray; BiONPs, bismuth oxide nanoparticles; Cis, cisplatin; BC, the combination of BiONPs and Cis.

only (control), both $\alpha$ and $\beta$ values are positive, indicating the double-strand breakage of DNA occurred by both single and double hits. ${ }^{13}$

Theoretically, when Ir-192 source is being used with the presence of nanoparticles with $Z$ of 20 to 90 , radiation doses could be enhanced, and the calculated DEF should be around 1.005 to $1.035 .{ }^{32}$ Conforming with a Monte Carlo study of Ir192 brachytherapy in the presence of $3 \%$ cisplatin in water, DEF of 1.07 was estimated. ${ }^{8}$ In the present study, it is found that the theoretical DEF for the individual BiONPs and Cis are different from both studies but can be considered consistent as it within the $10 \%$ difference. $^{32}$ The variation of DEF may be due to the disparity of nanoparticles concentration that was used, as emphasized by Taha et al. ${ }^{15}$ In conjunction

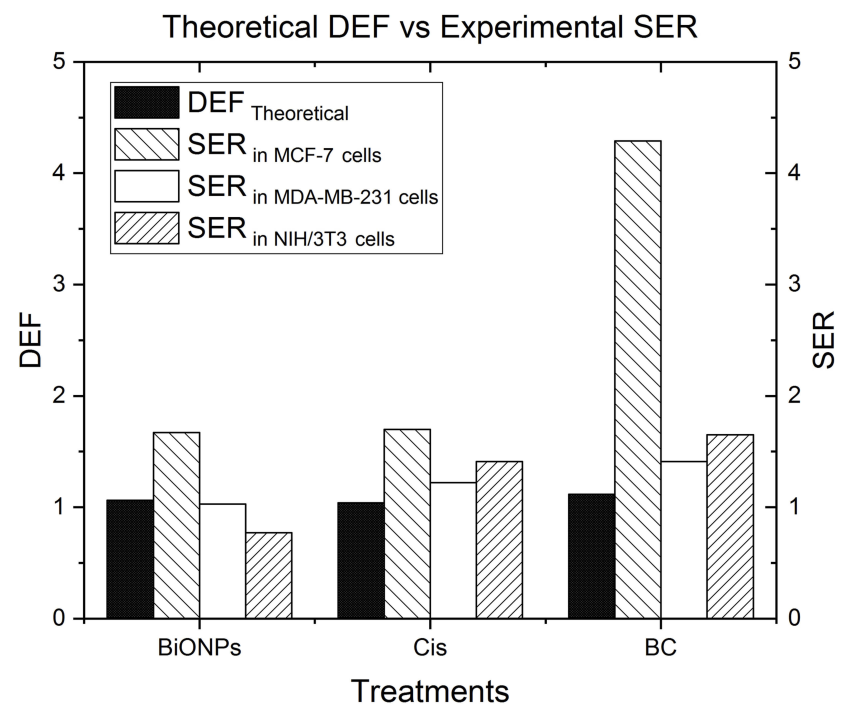

Figure 6 Comparison of theoretical DEF and experimental SER in MCF-7, MDAMB-23I, and NIH/3T3 cells.

Note: Theoretical DEF values are obtained from Figure I.

Abbreviations: DEF, dose enhancement ratio; SER, sensitization enhancement ratio; $\mathrm{BiONPs}$, bismuth oxide nanoparticles; Cis, cisplatin; BC, BiONPs-Cis combination. with the theoretical DEF, all the experimental SER observed in MCF-7 cells are higher than predicted values, notably when $\mathrm{BC}$ combinations were present during the irradiation. Such deviations may validate the involvement of chemical reactions and biological complex processes in the cell death mechanisms. ${ }^{10,11}$ Brun and Sicard-Roselli have summarized a few biological effects in radiosensitization, which may include the induction of radiosensitive phase of cell cycle such as G2/M, DNA repair damage concerning $\gamma-\mathrm{H} 2 \mathrm{AX}$ foci, initiation of endoplasmic reticulum stress, and overproduction of ROS. ${ }^{33}$

On the other hand, the experimental SER estimated in MDA-MB-231 cells with the presence of BiONPs is lower than expected. These results can be justified as MDA-MB231 cells were verified to be radioresistant. ${ }^{34,35}$ Current studies that investigated the molecular aspects of underlying radioresistance of cells had mentioned several mechanisms of action which involved the activation of WNT and Notch-1 pathways, ${ }^{34,36}$ as well as the expression of Jagged-1 ligand, ${ }^{36}$ Chk-1 kinases, ${ }^{35}$ XIAP apoptosis inhibitor, ${ }^{9}$ and $E R \alpha, P g R$ and $E G F R$ cell receptors. ${ }^{34} \mathrm{Up}$ and down-regulation of proapoptotic genes such as $F A D D, D A P K 1, C A S P 8, B A G-4$, CASP10, NOD1, and TNFSF10 were also affected in radioresistance of cells. ${ }^{9}$ However, the experimental SER, which is higher than 1, indicates increasing cell death from radiosensitization effects. The value suggested that there should be physical interactions between the $\mathrm{Cis}, \mathrm{BC}$, and radiation that could relegate the radioresistance and improve the radiosensitization towards the MDA-MB-231 cells.

Meanwhile in NIH/3T3 normal cells, the experimental SER is higher than theoretical DEF when BC combination was present during the irradiation, indicates that the healthy tissues surrounding the cancer site could be susceptible to the radiosensitization concerning the physical, 

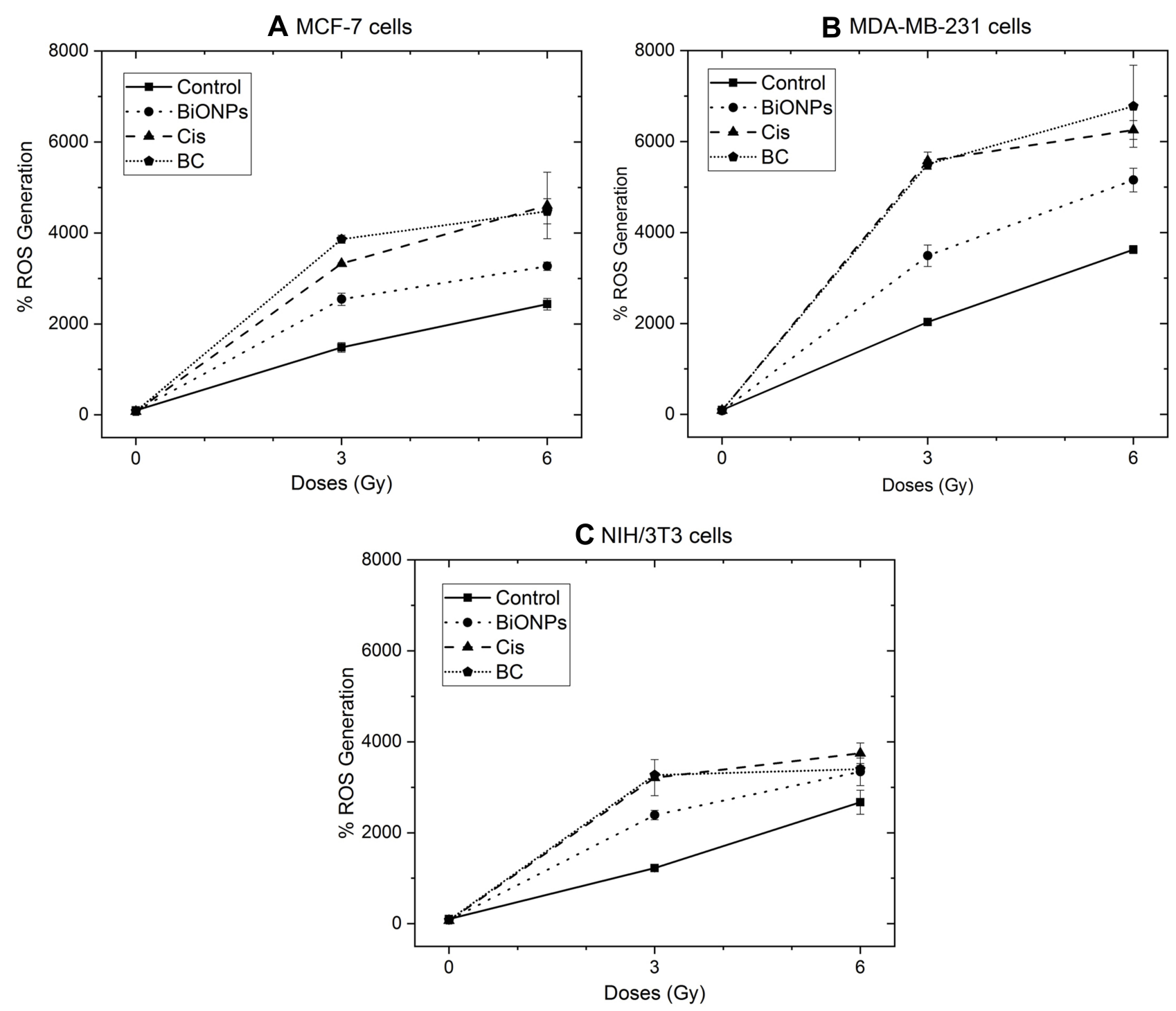

Figure 7 Reactive oxygen species (ROS) generation on (A) MCF-7, (B) MDA-MB-23I and (C) NIH/3T3 cells that underwent brachytherapy irradiation in the presence of BiONPs, Cis, and BC.

Notes: Error bars represent the errors of survival fractions.

chemical, and biological pathways. Though, this toxicity effects may be as negligible as side effects of concurrent chemoradiotherapy from clinical trials conducted. In a chemobrachytherapy study follow up after eight weeks of treatment, rectal, bladder and vaginal toxicities such as cystitis, dysuria, proctitis, and vaginal dryness, were found higher than in brachytherapy alone, yet they were considered insignificant. ${ }^{3}$ Another randomized clinical trial on brachytherapy and cisplatin demonstrated a few renal and hematological toxicities, but they were deemed acceptable. ${ }^{37}$ Conclusively Corde et al had explained that the difference in theoretical DEF and experimental SER could occur due to four factors: (1) different survival rate level, (2) exclusion of experimental variable conditions in theoretical simulation, (3) dissimilarity of energy division for cell killings in theoretical, in vitro and in vivo, and (4) variables of experimental conditions and sets up. ${ }^{38}$

Furthermore, ROS was known to exist in normal cells as well as in cancer cells. However, the induction of ROS in this study is more susceptible to cancer cells. ${ }^{39}$ This inclination is particularly true in regards to the substantial differences in the increment of intracellular ROS between cancer and normal cells after irradiation, as shown in Figure 7. When compared to intracellular ROS level without radiotherapy, the amount of ROS in MCF-7 and MDA-MB-231 cells were higher than that in NIH/3T3 cells by $263 \%$ and $812 \%$ respectively, after the cells were treated only with radiation of $3 \mathrm{~Gy}$ dose. When irradiated with a dose of $6 \mathrm{~Gy}$ in the presence of 
BC, the total rise of ROS in MCF-7 and MDA-MB-231 cells had increased by $1077 \%$ and $3378 \%$, respectively, when compared to NIH/3T3 cells. The intracellular ROS in cancer cells was considered vital as it is one of the indirect methods to reduce the survival rate of cancer cells. ${ }^{39}$ Apart from existed ROS in the cells, irradiation could cause water molecules to be lysed and formed various ROS. ${ }^{39}$ These ROS could preserve in the cells for a long time up to few months, in return disturbing the mitochondrial functions and DNA in the cells. ${ }^{39,40}$

The involvement of other compound or agent during cancer therapy had proven to instigate cells necrosis or apoptosis due to ROS contribution. ${ }^{41-43}$ Previous reports had observed that individual BiONPs or Cis could induce oxidative stress in cells. ${ }^{23,31,44}$ The BiONPs and Cis in the present study were determined to have an impact in escalating the ROS capacity during radiotherapy. The amount of ROS generated after irradiation were discovered to be cell-type- and dose-dependent. It also had been found that ROS had been boosted in the presence of the BiONPs and Cis in the following manner; control (radiation only), BiONPs, Cis, and then BC. However, this arrangement is only valid for MDA-MB-231 cells at 6 Gy dose irradiation and another two cells at 3 Gy dose irradiation.

In conjunction, it is perceived that there was no substantial difference between the percentage of ROS generated, in the presence of $\mathrm{Cis}$ and $\mathrm{BC}$. This finding is inconsistent with the sensitization enhancement measured formerly in this study. The BC combination might not have synergistic interaction in ROS induction as hypothesized in the SER part but instead formed antagonism. As stated by Brun et al, $\mathrm{DCFH}_{2}$-DA reagent could differentiate neither the types of ROS produced nor the sources of ROS production. ${ }^{33}$ In the present study, the main types of ROS produced by both BiONPs and Cis might be of different types, and the primary radicals materialized from each source might block the others. Also, there might be chemical reactions when BiONPs is combined with Cis. The proposed chemical reactions have yet to be explored. A few investigations had addressed the radicals scavenging effect by several bismuth complexes. Bismuth (III) salts could cause enhancement in hydroxyl radical scavenging activity when combined with acid-reducing drugs. ${ }^{45}$ Another study found the bismuth subnitrate was capable of decreasing oxidative stress caused by aluminum sulfate. ${ }^{46}$ An in vivo inquiry revealed that bismuth (III) selenide was able to reduce DNA damage after 6.5 Gy gamma rays irradiation. ${ }^{47}$ The latest research on biogenic bismuth NPs determined to express a moderate antioxidant activity, tested using DPPH and reducing power assays. ${ }^{48}$

Generally, sensitization enhancement measured previously conflicted with the ROS generation results. However, it is also known that many factors are affecting cellular damages in radiotherapy, as ROS-induced cell death is only one of the indirect methods. ${ }^{49}$ Even apoptosis and mitotic cell detach were not the primary mechanisms in radiotherapy efficiency. ${ }^{50}$ Therefore, in this study, ROS generation is considered as a secondary factor to the sensitization enhancement.

\section{Conclusion}

This study is the first precedent to experimentally evaluate the potential of the triple combination encompassing $\mathrm{BC}$ as a radiosensitizer and ionizing radiation in improving the current clinical radiotherapy for breast cancer treatment. BiONPs are found to be non-toxic and could be internalized by the cells. $\mathrm{BC}$ combination produces the highest radiosensitization especially for MCF-7 cells, with a fourfold increase compared to radiation treatment only. The presence of BiONPs and cisplatin also elevate the production of ROS in the cells. Interestingly, the NIH/3T3 cell which is the normal cell did not show high radiosensitization and ROS generation in comparison to the breast cancer cells. Future treatment with $\mathrm{BC}$ combination as a radiosensitizer in combination with HDR brachytherapy is considered as a harmless treatment approach to the healthy normal tissue and affecting the cancer cells only.

\section{Acknowledgments}

This work was supported by Universiti Sains Malaysia Research University Grant (RUI: 1001/PPSK/8012212) and Short Term Grant (304/PPSP/635121). We would like to convey our gratitude to the Nuclear Medicine, Radiotherapy and Oncology Department, Hospital Universiti Sains Malaysia, and the Central Research Laboratory, School of Medical Sciences, Universiti Sains Malaysia for the supports during experimental works.

\section{Disclosure}

The authors report no conflicts of interest in this work.

\section{References}

1. International Atomic Energy Agency (IAEA). Radiotherapy in cancer care: facing the global challenge; 2017. Available from: https:/www-pub. iaea.org/MTCD/Publications/PDF/P1638_web.pdf. Accessed December 9,2019 
2. Rahman WNWA. Gold nanoparticles: novel radiobiological dose enhancement studies for radiation therapy, synchrotron based microbeam and stereotactic radiotherapy [dissertation]. PhD. RMIT University; 2010.

3. Mukherjee A, Patra NB, Manir KS, Shyamal KS. A prospective clinical study on the benefit of adding chemotherapy to brachytherapy in patients with incomplete response to external beam irradiation with concurrent chemotherapy in locally advanced cases of carcinoma cervix. Indian J Med Res Pharm Sci. 2016;3(7):9-19. doi:10.5281/zenodo.1019549

4. Aghili M, Andalib B, Moghaddam ZK, Safaie M, Hashemi FA, Darzikolaie NM. Concurrent chemo- radiobrachytherapy with cisplatin and medium dose rate intra- cavitary brachytherapy for locally advanced uterine cervical cancer. Asian Pacific J Cancer Prev. 2018;19:2745-2750. doi:10.22034/APJCP.2018.19.10.2745

5. Chandel SS, Jain RK. Evaluation of role of radical radiotherapy and chemotherapy in cervical cancer patients- A preliminary report. Clin Cancer Investig J. 2016;2(5):126-131. doi:10.4103/2278-0513.177 127

6. Amouzegar-Hashemi F, Akbari EH, Esmati E. Concurrent chemoradiation with weekly gemcitabine and cisplatin for locally advanced cervical cancer. Asian Pacific J Cancer Prev. 2013;14:5385-5389. doi:10.7314/apjcp.2016.17.s3.287

7. Cui L, Her S, Dunne M, et al. Significant radiation enhancement effects by gold nanoparticles in combination with cisplatin in triple negative breast cancer cells and tumor xenografts. Radiat Res. 2017;187(2):147-160. doi:10.1667/RR14578.1

8. Cho S, Jeong JH, Kim CH, Yoon M. Monte Carlo simulation study on dose enhancement by gold nanoparticles in brachytherapy. $J$ Korean Phys Soc. 2010;56(6):1754-1758. doi:10.3938/jkps.56.1754

9. Gupta S, Koru-Sengul T, Arnold SM, Devi GR, Mohiuddin M, Ahmed MM. Low-dose fractionated radiation potentiates the effects of cisplatin independent of the hyper-radiation sensitivity in human lung cancer cells. Mol Cancer Ther. 2011;10(2):292-302. doi:10. 1158/1535-7163.mct-10-0630

10. Cui L Optimization of gold nanoparticle radiosensitizers for cancer therapy optimization of gold nanoparticle radiosensitizers [dissertation]. PhD. University of Toronto; 2016.

11. Rahman WN, Corde S, Yagi N, Abdul Aziz SA, Annabell N, Geso M. Optimal energy for cell radiosensitivity enhancement by gold nanoparticles using synchrotron-based monoenergetic photon beams. Int J Nanomedicine. 2014;9(1):2459-2467. doi:10.2147/IJN. S59471

12. Lazim RM, Rashid RA, Pham BTT, Hawkett BS, Geso M, Rahman WN. Radiation dose enhancement effects of superparamagnetic iron oxide nanoparticles to the T24 bladder cancer cell lines irradiated with megavoltage photon beam radiotheray. J Sains Nukl Malaysia. 2018;30(2):30-38.

13. Abdul Rashid R, Zainal Abidin S, Khairil Anuar MA, et al. Radiosensitization effects and ROS generation by high $\mathrm{Z}$ metallic nanoparticles on human colon carcinoma cell (HCT116) irradiated under $150 \mathrm{MeV}$ proton beam. OpenNano. 2019;4:100027. doi:10.1016/j.onano.2018.100027

14. Yang C, Bromma K, Sung W, Schuemann J, Chithrani D. Determining the radiation enhancement effects of gold nanoparticles in cells in a combined treatment with cisplatin and radiation at therapeutic megavoltage energies. Cancers (Basel). 2018;10(150):116. doi:10.3390/cancers 10050150

15. Taha E, Djouider F, Banoqitah E. Monte Carlo simulations for dose enhancement in cancer treatment using bismuth oxide nanoparticles implanted in brain soft tissue. Australas Phys Eng Sci Med. 2018;41:363-370. doi:10.1007/s13246-018-0633-z

16. Stewart C, Konstantinov K, McKinnon S, et al. First proof of bismuth oxide nanoparticles as efficient radiosensitisers on highly radioresistant cancer cells. Phys Medica. 2016;32:11. doi:10.1016/j.ejmp. 2016.10.015
17. Stewart CAC An investigation into the tailoring of bismuth oxide nanoceramic with a biomedical application as a high $\mathrm{Z}$ radiation enhancer for cancer therapy [dissertation]. Master of Science. University of Wollongong; 2014.

18. Ovsyannikov VA, Zamoryanskaya MV, Semencha AV, et al. Development of bismuth oxide-based nanopreparation for the destruction of malignant neoplasms: theoretical prerequisites, challenges, and practical approaches. Glas Phys Chem. 2015;41(5):533536. doi:10.1134/S1087659615050119

19. Zulkifli ZA, Razak KA, Rahman WNWA, Abidin SZ. Synthesis and characterisation of bismuth oxide nanoparticles using hydrothermal method: the effect of reactant concentrations and application in radiotherapy. J Phys Conf Ser. 2018;1082:1. doi:10.1088/1742-6596/1082/ $1 / 012103$

20. Alqathami M, Blencowe A, Geso M, Ibbott G. Quantitative 3D determination of radiosensitization by bismuth-based nanoparticles. J Biomed Nanotechnol. 2016;12(3):464-471. doi:10.1166/jbn.2016. 2183

21. Hubbell JH, Seltzer SM. X-ray mass attenuation coefficients. NIST Standard Reference Database 126. 2004; Available from: http://www. nist.gov/pml/x-ray-mass-attenuationcoefficients. Accessed March 18, 2019

22. Nor NAM, Mohd Z, Razak HRA, Eshak Z, Saad WMM. Synthetisation temperature-dependent cytotoxicity of bismuth oxide nanoparticles in vitro. Pertanika J Sci Technol. 2017;25(S):227-236.

23. Ahamed M, Akhtar MJ, Khan MAM, Alrokayan SA, Alhadlaq HA. Oxidative stress mediated cytotoxicity and apoptosis response of bismuth oxide (Bi2O3) nanoparticles in human breast cancer (MCF-7) cells. Chemosphere. 2019;216:823-831. doi:10.1016/j. chemosphere.2018.10.214

24. Feoktistova M, Geserick P, Leverkus M. Crystal violet assay for determining viability of cultured cells. Cold Spring Harb Protoc. 2016;2016(4):343-346. doi:10.1101/pdb.prot087379

25. Sisin NNT, Abidin SZ, Yunus MA, Zin HM, Razak KA, Rahman WN. Evaluation of bismuth oxide nanoparticles as radiosensitizer for megavoltage. Int J Adv Sci Eng Inf Technol. 2019;9(4):1434-1443. doi:10.18517/ijaseit.9.4.7218

26. Passagne I, Morille M, Rousset M, Pujalté I, L'Azou B. Implication of oxidative stress in size-dependent toxicity of silica nanoparticles in kidney cells. Toxicology. 2012;299(2-3):112-124. doi:10.1016/j. tox.2012.05.010

27. Kim TH, Kim M, Park HS, Shin US, Gong MS, Kim HW. Sizedependent cellular toxicity of silver nanoparticles. J Biomed Mater Res Part A. 2012;100A:1033-1043. doi:10.1002/jbm.a.34053

28. Boo L, Ho WY, Ali NM, et al. Phenotypic and microRNA transcriptomic profiling of the MDA-MB-231 spheroid- enriched CSCs with comparison of MCF- 7 microRNA profiling dataset. PeerJ. 2017;5 (e3551):1-27. doi:10.7717/peerj.3551

29. Carraher CE, Michael J, Ryan RR, Lindsey AM, Kendra M, Floyd B. Synthesis and preliminary cancer cell line results for the product of organotin dihalides and alpha-cyano-4-hydroxycinnamic acid. J Inorg Organomet Polym Mater. 2016;1-11. doi:10.1007/s10904-016-03631

30. Eblan MJ, Wang AZ. Improving chemoradiotherapy with nanoparticle therapeutics. Transl Cancer Res. 2013;2(4):320-329. doi:10.3978/ j.issn.2218-676X.2013.08.04

31. Ghosh P, Singh A, Roy S, Basu A, Bhattacharjee A, Bhattacharya S. Sensitization of cisplatin therapy by a naphthalimide based organoselenium compound through modulation of antioxidant enzymes and p53 mediated apoptosis. Free Radic Res. 2015;49(4):453-471. doi:10.3109/10715762.2015.1012079

32. Roeske JC, Nuñez L, Hoggarth M, Labay E, Weichselbaum RR. Characterization of the theorectical radiation dose enhancement from nanoparticles. Technol Cancer Res Treat. 2007;6(5):395-401. doi:10.1177/153303460700600504 
33. Brun E, Sicard-Roselli C. Actual questions raised by nanoparticle radiosensitization. Radiat Phys Chem. 2016;128:134-142. doi:10. 1016/j.radphyschem.2016.05.024

34. Gray M, Turnbull AK, Ward C, et al. Development and characterisation of acquired radioresistant breast cancer cell lines. Radiat Oncol. 2019;14(1):1-19. doi:10.1186/s13014-019-1268-2

35. Dinkelborg PH, Wang M, Gheorghiu L, et al. A common Chk1dependent phenotype of DNA double-strand break suppression in two distinct radioresistant cancer types. Breast Cancer Res Treat. 2019;174(3):605-613. doi:10.1007/s10549-018-05079-7

36. Phillips TM, McBride WH, Pajonk F. The response of CD24-/low/ CD44+ breast cancer-initiating cells to radiation. J Natl Cancer Inst. 2006;98(24):1777-1785. doi:10.1093/jnci/djj495

37. Aghili M, Andalib B, Hashemi FA, et al. Concurrent chemo-brachytherapy with cisplatin and intracavitary brachytherapy in locally advanced uterine cervical cancer. CANCER Bull Cancer Inst Iran. 2010;2:45-52.

38. Corde S, Joubert A, Adam JF, et al. Synchrotron radiation-based experimental determination of the optimal energy for cell radiotoxicity enhancement following photoelectric effect on stable iodinated compounds. $\mathrm{Br} \mathrm{J}$ Cancer. 2004;91(3):544-551. doi:10.1038/sj.bjc.6601951

39. Tong L, Chuang C, Wu S, Zuo L. Reactive oxygen species in redox cancer therapy. Cancer Lett. 2015;07:008. doi:10.1016/j.canlet.2015.07.008

40. Vlashi E, Pajonk F. Cancer stem cells, cancer cell plasticity and radiation therapy. Semin Cancer Biol. 2015;31:28-35. doi:10.1016/j. semcancer.2014.07.001.Cancer

41. Saeidnia S, Abdollahi M. Antioxidants: friends or foe in prevention or treatment of cancer: the debate of the century. Toxicol Appl Pharmacol. 2013;271:49-63. doi:10.1016/j.taap.2013.05.004

42. Sai S, Shirai T, Kamada T. Effects of carbon ion beam alone or in combination with Chk1 inhibitor and gemcitabine on putative pancreatic cancer stem cells. Pancreatology. 2013;13(4):S2. doi:10.1016/ j.pan.2013.07.066
43. Cheng K, Sano M, Jenkins CH, Zhang G, Vernekohl D. Synergistically enhancing therapeutic effect of radiation therapy with radiation activatable and reactive oxygen species-releasing nanostructures. ACS Nano. 2018;12(5):4946-4958. doi:10.1021/ acsnano. 8 b02038

44. Stewart C, Konstantinov K, McDonald M, et al. Engineering of bismuth oxide nanoparticles to induce differential biochemical activity in malignant and nonmalignant cells. Part Part Syst Charact. 2014;31(9):960-964. doi:10.1002/ppsc.201300380

45. Kirkova M, Alexandrova A, Yordanova N. Bismuth increases hydroxyl radical-scavenging activity of histamine H2-receptor antagonists. Pharmacol Rep. 2006;58(2):215-220.

46. Turkez H, Geyikoglu F. The efficiacy of bismuth subnitrate against genotoxicity and oxidative stress induced by aluminum sulphate. Toxicol Ind Health. 2011;27(2):133-142. doi:10.1177/07482337103 81894

47. Zhang XD, Jing Y, Song S, et al. Catalytic topological insulator $\mathrm{Bi} 2 \mathrm{Se} 3$ nanoparticles for in vivo protection against ionizing radiation. Nanomed Nanotechnol Biol Med. 2017;13(5):1597-1605. doi:10.10 16/j.nano.2017.02.018

48. Shakibaie M, Amiri-Moghadam P, Ghazanfari M, Adeli-Sardou M, Jafari M, Forootanfar H. Cytotoxic and antioxidant activity of the biogenic bismuth nanoparticles produced by Delftia sp. SFG. Mater Res Bull. 2018;104:155-163. doi:10.1016/j.materresbull.2018.04.001

49. Baskar R, Lee KA, Yeo R, Yeoh K-W. Cancer and radiation therapy: current advances and future directions. Int J Med Sci. 2012;9(3):193199. doi:10.7150/ijms.3635

50. Martins I, Raza SQ, Voisin L, et al. Anticancer chemotherapy and radiotherapy trigger both non-cell-autonomous and cell-autonomous death article. Cell Death Dis. 2018;9:7. doi:10.1038/s41419-0180747-y
International Journal of Nanomedicine

\section{Publish your work in this journal}

The International Journal of Nanomedicine is an international, peerreviewed journal focusing on the application of nanotechnology in diagnostics, therapeutics, and drug delivery systems throughout the biomedical field. This journal is indexed on PubMed Central, MedLine, CAS, SciSearch ${ }^{\mathbb{}}$, Current Contents ${ }^{\mathbb{R}} /$ Clinical Medicine,

\section{Dovepress}

Journal Citation Reports/Science Edition, EMBase, Scopus and the Elsevier Bibliographic databases. The manuscript management system is completely online and includes a very quick and fair peer-review system, which is all easy to use. Visit http://www.dovepress.com/ testimonials.php to read real quotes from published authors. 